\title{
MEMBERS OF THE COUNCIL
}

OfFICERS

Virgil SNyder, President.

E. T. BELL

O. D. KELLOGG

C. N. MOORE

Vice-Presidents.

R. G. D. Richardon, Secretary.

ARNold DRESDEN, Assistant Secretary.

W. B. Fite, Treasurer.

R. C. ARCHIBALD, Librarian.

E. R. HEDRICK ARNold DRESDEN

W. R. LONGLEY DUNHAM JACKSON

EDWARD KASNER

H. H. MrtCheLl

Bulletin Editorial Committee.

Transactions Editorial Committee.

\section{Ex-Presidents}

G. A. Bliss,

Oswald Veblen,

G. D. Birkhoff.

\section{Elected Members}

To serve until December, 1927
Daniel Buchanan,
E. W. Chitrenden,
G. A. Campbell,
A. J. KEMPNER,

H. E. Slaught.

To serve until December, 1928
$\begin{array}{ll}\text { Tomlinson ForT, } & \text { J. F. RitT, } \\ \text { Olive C. HAZLETT, } & \text { E. B. Stouffer, }\end{array}$
Norbert WiENER.

To serve until December, 1929

A. A. Bennetr,

J. W. GLOVER,

JAMES Pierpont,

G. E. WAhlin.

H. S. VANDIVER,

\section{BOARD OF TRUSTEES}

1927

W. B. Fite,

Robert Henderson,

R. G. D. RichaRdSON,

VIRGIL SNYDER,

$$
\text { Óswald Veblen. }
$$

